\title{
ASSESSMENT OF THE RELATIONSHIP BETWEEN PERIODONTAL DISEASE AND CARDIOVASCULAR DISORDERS: A QUESTIONNAIRE-BASED STUDY
}

\author{
ANCA IONEL ${ }^{1}$, ONDINE LUCACIU ${ }^{1}$, COSMINA BONDOR ${ }^{2}$, \\ MINODORA MOGA ${ }^{1}$, ARANKA ILEA ${ }^{1}$, CLAUDIA FEURDEAN ${ }^{1}$, \\ DAN BUHĂȚEL ${ }^{1}$, LUCIA HURUBEANU ${ }^{3}$, RADU SEPTIMIU CÂMPIAN ${ }^{1}$
}

\author{
${ }^{1}$ Department of Oral Rehabilitation, Oral Health and Dental Office Management, \\ Iuliu Hatieganu University of Medicine and Pharmacy, Cluj-Napoca, Romania \\ ${ }^{2}$ Department of Medical Informatics and Biostatistics, Iuliu Hatieganu University \\ of Medicine and Pharmacy, Cluj-Napoca, Romania \\ ${ }^{3}$ Department of Oral and Cranio-Maxillofacial Surgery, Iuliu Hatieganu \\ University of Medicine and Pharmacy, Cluj-Napoca, Romania
}

\begin{abstract}
Background and aims. Periodontitis is an inflammatory disease, characterized by the loss of connective tissue and alveolar bone. There is an increasing evidence that periodontitis is associated with a number of chronic diseases. The aim of this study was to analyze the correlation between periodontitis and certain systemic diseases by identifying their risk factors in a population from North-West Romania.

Methods. A questionnaire-based study was conducted on participants $\geq 45$ years of age in 4 dentistry or family medicine practices from the Cluj and Bihor counties, Romania, over a time frame spanning two months. The intervieweradministered questionnaire included 42 items assessing risk factors for periodontal and cardiovascular disease, current diagnostic and medical treatment of the participants, their diet and oral hygiene habits.

Results. Of 108 participants included in the study, 63 (58.3\%) declared a previous diagnosis of periodontitis. A significant difference was observed between the participants with declared diagnosis of periodontitis $(P D+)$ and participants without declared diagnosis of periodontitis (PD-) for fresh fruit consumption $(p=0.01)$ and increased sport activity $(p=0.009)$. A significant difference between groups was observed in the case of smoking duration ( $p=0.047)$, participants with $>10$ years of smoking were more likely to declare a diagnosis of periodontitis. Also, $P D+$ participants were more likely to have a family history of periodontal disease $(p<0.001)$. Multivariate analyses suggested periodontitis as a predictive variable for atherosclerotic coronary artery disease with an OR of 4.85 (95\% confidence interval 1.01-23.29, $p=0.049)$.

Conclusion. Our study found a statistically significant association between periodontal disease and a known family history of periodontal disorders and smoking duration. Daily intake of fresh fruit and increased sport activities were found to be protective factors against periodontal disease, while periodontitis was found as a risk factor for atherosclerotic cardiovascular disease.
\end{abstract}

Keywords: periodontitis, systemic diseases, questionnaire, oral health

Manuscript received: 27.01.2016

Received in revised form: 24.03 .2016

Accepted: 29.03.2016

Address for correspondence: ondineluc@yahoo.com 


\section{Background and aims}

Periodontal disease is a localized, chronic, inflammatory disease which results in loss of connective tissue and bone support, being the main cause of tooth loss in adults [1]. Periodontal disease coupled with systemic implications and metabolic disorders is highly prevalent among the population and their correlation could have an impact on the lives of many people. The association between them is the subject of research, and a variety of theories have been proposed, though most of them postulate the mediation of an inflammatory response [2]. This bi-directional relation between periodontitis and systemic disease such as diabetes, autoimmune and cardiovascular diseases is suggested by the fact that oral infection is a constant potential source for general infection and inflammation $[3,4]$. On the other hand, systemic factors that affect the overall condition of the body can have a negative influence on periodontal tissues. It is assumed that systemic factors themselves cannot cause an inflammatory local response. However, these factors may play a role in the etiology of periodontal disease by modifying the body immune response to periodontal bacteria and their products [5].

Multiple epidemiological studies have found periodontal disease an independent risk factor for cardiovascular diseases starting from the premise that local infection can affect systemic inflammatory mediators, thus leading to atherosclerosis and coronary complications [6].

Periodontal disease and cardiovascular disease also share a common set of risk factors: the male gender, smoking, alcohol consumption, stress, certain infectious agents and age. The relationship between periodontal disease and cardiovascular disease is very complex and at present not well defined. Thus, there is a need to continue studies based on the pathogenesis and diagnosis of periodontal disease and to elaborate novel methods of treating this disease ultimately aiming at impacting the general health of the patient [7].

There is evidence indicating a direct relationship between periodontal disease and diabetes mellitus, and also evidence indicating a higher prevalence of diabetes in patients with periodontitis compared to patients without the disease [8]. Epidemiological studies showed that the risk of alveolar bone loss and attachment loss is higher in diabetic patients compared to non-diabetic individuals [9].

Besides the fact that obesity is considered a risk factor for cardiovascular disease, several types of cancer and diabetes mellitus, it has also been suggested to be a risk factor for periodontitis [3]. The exact mechanism by which obesity has an effect on periodontitis is not completely understood, but it is thought to be mediated by the altered insulin sensitivity state, increased local inflammatory response or by the secretion of different bioactive compounds secreted by the adipose tissue [10].

The high prevalence of chronic periodontitis and systemic diseases - particularly atherosclerosis manifesting as ischemic coronary disease or diabetes mellitus - as major risk factors for public health causing increasing rates of morbidity and mortality leads to an increased interest for new research of their relationship. Thus, the current study aims at analyzing the correlation between periodontal disease and systemic diseases by identifying existing correlations and their risk factors in a population from North-West Romania, through an interviewer-administered questionnaire.

\section{Materials and Methods Study design}

This was a questionnaire-based study administered to adults over 45 years of age. Participants enrolled in the study came in for a consultation to 4 different dentistry or family medicine practices in counties of Cluj and Bihor, Romania, over a time frame spanning two months.

Data was collected by administering the study questionnaire as a structured interview by 6 investigators who were trained regarding data entry before the study started. Study participants were included by non-probability sampling, consecutive selection. All study participants gave informed consent to participation and use of data. The study was approved by Medical Ethics Committee of Iuliu Hatieganu University of Medicine and Pharmacy, ClujNapoca .

\section{The questionnaire}

The questionnaire items included risk factors for periodontal and systemic diseases and the link between them. The questionnaire was composed of two sections: 1) participant demographic characteristics and 2) one base section referring to periodontal disease, systemic disease, risk factors and lifestyle habits.

The questionnaire contained 42 items and was structured as follows: 4 items dealing with the declared diagnosis (periodontal disease, cardiovascular disease and diabetes mellitus); 13 items investigating the presence or absence of risk factors for periodontitis, diabetes and cardiovascular disease; 8 items looking into the presence or absence of signs and symptoms for disease; 2 items dealing with the timeframe in which the pathology appeared; 6 items investigating current medical treatment; 6 items dealing with food consumption, diet and physical activity of the participants and finally 3 items assessing oral health and hygiene. The questionnaire contained 18 closed questions with „Yes”, „No” and „I don’t know” possible answers, 14 multiple-choice questions and 10 open ended questions. The evaluation of physical activity habits and nutritional habits was performed using a scale from 0 to $4(0=$ never; 1=1 times/week; 2=2-3 times/week; 3=4-6 times/week; $4=$ daily). Four experts (dental medicine and statistics) were consulted and the final questionnaire was created based on their input. A pilot study was undertaken on 10 participants to evaluate the clarity, relevance, simplicity and coherence of the study questionnaire. All 10 participants described the 


\section{Dental Medicine}

questionnaire as open and easy to understand. Finally, the questionnaire was validated by the test-retest method; 30 volunteers filled in the questionnaire twice at an interval of 2 weeks. For the questions with two possible answers a Cohen's $\kappa$ coefficient of agreement of $0.86-1$ was obtained, while for the questions with multiple possible answers the Cronbach $\alpha$ value was between $0.87-1$.

\section{Statistical analysis}

Data obtained from the questionnaires was introduced in Microsoft Excel. All „I don’t know” answers were encoded as a false response. Statistical analysis was performed using SPSS Statistics for Windows, Version 17.0 (SPSS Inc., Chicago, USA). Qualitative variables were analyzed using the Chi-squared test and quantitative variables were analyzed with the $\mathrm{T}$ test and Mann-Whitney test.

Two multivariate logical regression analyses (forward LR method) were performed. The first used periodontitis as dependent variable and the following parameters as independent variables: gender, area (rural versus urban), age, arterial hypertension, diabetes mellitus, hypercholesterolemia, smoking, alcohol consumption, fresh fruit and vegetables consumption, dairy product consumption, consumption of sweets and sodas, physical activity, body mass index (BMI), systolic blood pressure, diastolic blood pressure, frequency of dental hygiene, frequency of dental specialist visits, declared diagnosis of ischemic heart disease, angina pectoris, myocardial infarction, atherosclerotic coronary disease, stroke and cardiac insufficiency. The second was performed for each of the following variables dependent on cardiovascular disease: ischemic heart disease, angina pectoris, myocardial infarction, atherosclerotic coronary disease, stroke, heart failure. The following were used as independent predictive variables: gender, age, rural vs. urban, arterial hypertension, diabetes mellitus, hypercholesterolemia, smoking, alcohol consumption, fresh vegetables and fruits consumption, milk product consumption, sweets and sweetened-beverage consumption, amount of physical activity, body mass index, systolic blood pressure (SBP), diastolic blood pressure (DBP), frequency of brushing teeth, frequency of visiting a dentist and declared diagnosis of periodontal disease. Both these analyses were performed on participants who had answered all questions related to the variables.

\section{Results}

A total of 108 participants were included in the study. The average age $( \pm \mathrm{SD})$ was $55.74 \pm 10.51$ years, women represented $55 \%$ of the study group and $52.8 \%$ of participants came from an urban area, showing a balanced study group. Previous diagnosis of periodontal disease was declared by 63 participants $(58.3 \%)$.

Statistical analysis regarding demographic parameters in subgroups of participants who declared a diagnosis of periodontal disease $(\mathrm{PD}+)$ or did not declare a diagnosis of periodontal disease (PD-) is given in Table I. No statistically significant difference was obtained between the groups regarding average age, gender, urban vs. rural or educational level. Table I also shows age, BMI, diastolic and systolic blood pressure, last known glycemia and cholesterol values as well as the number of absent teeth in the PD+ and PD- subgroups. No significant differences were observed between groups, except when analyzing the number of absent teeth $(\mathrm{p}<0.001)$.

A statistically significant difference was observed between the $\mathrm{PD}+$ and $\mathrm{PD}$ - groups in the case of fresh fruit consumption $(\mathrm{p}=0.01)$ and increased sport activity $(\mathrm{p}=0.009)$.

Oral hygiene habits and the frequency of visiting a dentist were also analyzed using the questionnaire (Table II). Twenty seven per cent of PD+ participants declared brushing once daily, $52.4 \%$ twice daily, while $9.5 \%$ declared that they did not brush their teeth every day. Results were similar in the PD- group ( $\mathrm{p}=0.44)$. The frequency of dental practitioner visits was also similar in the $\mathrm{PD}+$ and $\mathrm{PD}-$ groups $(\mathrm{p}=0.89)$, with a numerical excess of participants in the $\mathrm{PD}+$ group only visiting a dental practitioner for emergencies ( $40 \%$ versus $28 \%$ in the PD+ and PD- groups respectively).

More than half of participants in each subgroup were smokers or former smokers (25.4\% smokers and $25.4 \%$ exsmokers in $\mathrm{PD}+, 28.8 \%$ smokers and $31.1 \%$ ex-smokers in PD-), but the difference was not statistically significant $(\mathrm{p}=0.51)$. A statistically significant difference between groups was observed in the case of duration of smoking $(\mathrm{p}=0.047$, Figure 1 ), with participants with $>10$ years of smoking more likely to declare a diagnosis of periodontal disease.

A significant difference between the $\mathrm{PD}+$ and PD- groups was observed in terms of a family history of periodontal disease $(p<0.001)$. Thus, $93.5 \%$ of participants in the PD+ subgroup declared a family history of periodontal disease, while only $32.2 \%$ of the participants in the PDsubgroup answered the question positively.

Periodontal disease symptoms (gingival bleeding, dental mobility and gingival retractions) were significantly more often declared by participants in the PD+ subgroup compared to the PD- subgroup (Table III).

For participants in the PD- group, the presence of symptoms was further analyzed: $35.6 \%$ declared having both gingival bleeding and retractions, $8.9 \%$ only gingival retractions, $22.2 \%$ only bleeding, while $33.3 \%$ declared having none of these symptoms. A statistically significant relationship was found between these symptoms $(p=0.007)$.

The presence of edentulism was found to be significantly associated with periodontal disease $(\mathrm{p}=0.015)$. In the PD+ subgroup, all participants had edentulism, while these were declared by $90.9 \%$. The number of absent teeth was also significantly different between groups $(p=0.003)$. Thus, all participants with complete edentulism had a 
previous declared diagnosis of periodontal disease and a higher number of participants in the PD + subgroup had more than 10 missing teeth. The lack of edentulism was only declared by participants from the PD- subgroup (Figure 2).

Diabetes was declared by $36.5 \%$ of participants in the PD+ subgroup and by $42.2 \%$ in the PD- subgroup, while cardiovascular disease was declared by $38.1 \%$ and $35.6 \%$ in the PD+ and PD- subgroups, respectively. A total of $66.0 \%$ participants declared a family history of cardiovascular disease and $35.8 \%$ a family history of diabetes in the PD+ subgroup, while the respective values were $80.0 \%$ and $66.7 \%$ in the PD- subgroup. No significant difference was observed between groups in the case of declared systemic diseases or family history of systemic diseases such as diabetes or cardiovascular disease.
Arterial hypertension was also not found to be significantly associated with a known declared diagnosis of periodontal disease $(\mathrm{p}=0.135)$, with $61.9 \%$ and $75.6 \%$ of participants from the PD+ and PD- subgroups, respectively, declaring the presence of arterial hypertension. Similarly, periodontal disease was not associated with hypercholesterolemia $(\mathrm{p}=0.30)$.

Although among the participants in this study hypercholesterolemia was significantly associated with cardiovascular disease presence $(\mathrm{p}=0.03)$, there was no significant association between hypercholesterolemia and periodontal disease $(\mathrm{p}=0.30)$, with $52.4 \%$ and $62.2 \%$ of participants in the PD+ and PD- subgroups, respectively, declaring hypercholesterolemia.

Table I. Participant characteristics in groups with or without a previous declared diagnosis of periodontal disease.

\begin{tabular}{|c|c|c|c|c|}
\hline \multicolumn{2}{|c|}{ Parameter } & $\begin{array}{c}\begin{array}{c}\text { PD+ } \\
(\mathrm{N}=63)\end{array} \\
\end{array}$ & $\begin{array}{c}\text { PD- } \\
(\mathrm{N}=45)\end{array}$ & $\mathbf{p}$ \\
\hline \multicolumn{5}{|c|}{ Demographic parameters, average \pm SD } \\
\hline Average age, (years \pm SD) & & $57.17 \pm 10.52$ & $53.73 \pm 10.56$ & 0.09 \\
\hline Area, urban $(\%)$ & urban & 49.2 & 57.8 & 0.39 \\
\hline Gender, male (\%) & male & 41.3 & 51.1 & 0.31 \\
\hline \multirow{3}{*}{ Educational level (\%) } & middle school & 14.3 & 15.6 & \multirow{3}{*}{0.57} \\
\hline & high school & 57.1 & 64.4 & \\
\hline & university & 28.6 & 20.0 & \\
\hline \multicolumn{5}{|c|}{ Clinical parameters, average \pm SD/median ( 25 th- 75 th percentile) } \\
\hline & BMI & $\begin{array}{c}26.56(24.49- \\
30.64)\end{array}$ & $\begin{array}{c}27.78(25.37- \\
32.37)\end{array}$ & 0.24 \\
\hline & $\mathrm{SBP}(\mathrm{mmHG})$ & $139(120-150)$ & $130(120-150)$ & 0.32 \\
\hline & $\mathrm{DBP}(\mathrm{mmHG})$ & $90(80-90)$ & $85(80-90)$ & 0.18 \\
\hline & Serum total cholesterol (mg/dl) & $209.77 \pm 56.97$ & $210.07 \pm 39.41$ & 0.98 \\
\hline \multirow{5}{*}{$\begin{array}{l}\text { Number of absent teeth, } \\
\mathrm{n}(\%)\end{array}$} & no absent teeth & $0(0.0)$ & $4(8.9)$ & $<0.001$ \\
\hline & $1-5$ & $12(26.7)$ & $12(19.0)$ & \\
\hline & $5-10$ & $13(20.6)$ & $14(31.1)$ & \\
\hline & over 10 & $27(42.9)$ & $15(33.3)$ & \\
\hline & all & $11(17.5)$ & $0(0.0)$ & \\
\hline \multicolumn{5}{|c|}{ Lifestyle habits, median score (25th -75 th percentile) } \\
\hline \multirow{4}{*}{ Dietary habits } & Fresh vegetable consumption & $2(2-3)$ & $3(2-4)$ & 0.33 \\
\hline & Fresh fruit consumption & $2(1-3)$ & $3(2-4)$ & 0.01 \\
\hline & Consumption of dairy products & $2(2-3)$ & $2(1.5-3)$ & 0.99 \\
\hline & Consumption of sweets or sodas & $1(1-2)$ & $1(1-2)$ & 0.37 \\
\hline \multirow[t]{2}{*}{ Physical effort } & $\begin{array}{c}\text { Physical effort during day to } \\
\text { day activities }\end{array}$ & $2(1-2)$ & $2(1-2.5)$ & 0.85 \\
\hline & Sport activities & $0(0-1)$ & $1(0-1.5)$ & 0.009 \\
\hline
\end{tabular}

$\mathrm{PD}+$, participants with a declared periodontal disease diagnosis; PD-, participants without a declared periodontal disease diagnosis; DBP, diastolic blood pressure; SBP, systolic blood pressure; SD, standard deviation. 
For participants who declared a known periodontal disease diagnosis and who also had cardiovascular disease, the time since the diagnosis was also analyzed. $41.5 \%$ of participants declared that periodontal disease was the first diagnosis, $51.2 \%$ declared a diagnosis in a close time frame, while $7.3 \%$ declared that cardiovascular disease was diagnosed earlier. Out of all participants who declared a known periodontal disease diagnosis before cardiovascular disease $(\mathrm{n}=17), 70.6 \%$ declared having arterial hypertension, $41.2 \%$ cardiopathic ischemia, $7.6 \%$ angina pectoris, $17.6 \%$ a previous myocardial infarction, $35.3 \%$ atherosclerotic coronary disease, $5.9 \%$ a previous stroke and $11.8 \%$ heart failure. For participants with cardiovascular disease diagnosed at a similar time with periodontal disease $(n=21)$,
95.2\% also had arterial hypertension, $38.1 \%$ cardiopathic ischemia, $9.5 \%$ angina pectoris, $14.3 \%$ a previous myocardial infarction, $23.8 \%$ atherosclerotic coronary disease, $4.8 \%$ a previous stroke and $14.3 \%$ cardiac failure.

Participants' responses regarding cardiovascular disease are presented in Table IV.

Multivariate analyses showed that fresh fruit consumption may be regarded as a protective factor for periodontitis with an odds ratio (OR) of 0.67 (95\% confidence interval $[\mathrm{CI}]$ 0.46-0.97, $\mathrm{p}=0.03)$. Also, in the present study, periodontitis was not a predictive variable for cardiovascular disease except for atherosclerotic coronary artery disease with an OR of 4.85 (95\% CI 1.01-23.29, $\mathrm{p}=0.049$ ).

Table II. Frequency of tooth brushing and visits to the dentist.

\begin{tabular}{lccc}
\hline & PD+ $(\mathbf{N}=\mathbf{6 3})$ & PD- $(\mathbf{N}=\mathbf{4 5})$ & $\begin{array}{c}\text { All participants } \\
(\mathbf{N}=\mathbf{1 0 8})\end{array}$ \\
\hline Brushing teeth/day, $\mathbf{n}$ & 6 & 5 & 11 \\
$<1$ & 17 & 12 & 29 \\
1 & 33 & 27 & 60 \\
2 & 7 & 1 & 8 \\
3 & & & \\
Visits to the dentist, $\mathbf{n}$ & 1 & 2 & 3 \\
Never & 10 & 3 & 13 \\
Every 6 months & 12 & 12 & 24 \\
Every year & 40 & 28 & 68 \\
Emergencies only & & & \\
\hline
\end{tabular}

$\mathrm{N}$, number of participants per group; $\mathrm{n}$, number of participants in a given category, $\mathrm{PD}+$, participants with a declared periodontal disease diagnosis present; PD-, participants without a declared periodontal disease diagnosis

\section{All smoker participants}

PD-

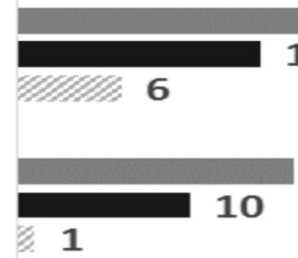

14

1

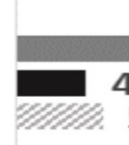

4
16

23

39

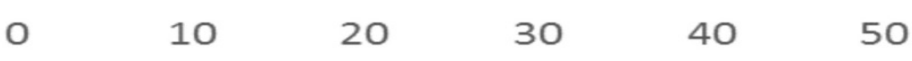

Percentage of smoker participants

Figure 1. Smoking duration in smoker and ex-smoker participants.

Footnote: $\mathrm{PD}+$, participants with a declared periodontal disease diagnosis present; PD-, participants without a declared periodontal disease diagnosis. 
Table III. Periodontal disease symptoms as declared by participants.

\begin{tabular}{l|c|c|c}
\hline Periodontal disease symptoms & $\begin{array}{c}\text { PD+ (N=63) } \\
\mathbf{\%}\end{array}$ & $\begin{array}{c}\text { PD- (N=45) } \\
\mathbf{\%}\end{array}$ & $\mathbf{p}$ \\
\hline Gingival bleeding & 76.2 & 57.8 & $\mathbf{0 . 0 4 2}$ \\
\hline Dental mobility & 52.4 & 17.8 & $<\mathbf{0 . 0 0 1}$ \\
\hline Gingival retractions & 81.0 & 44.4 & $<\mathbf{0 . 0 0 1}$ \\
\hline
\end{tabular}

$\mathrm{PD}+$, participants with a declared periodontal disease diagnosis present; PD-, participants without a declared periodontal disease diagnosis.

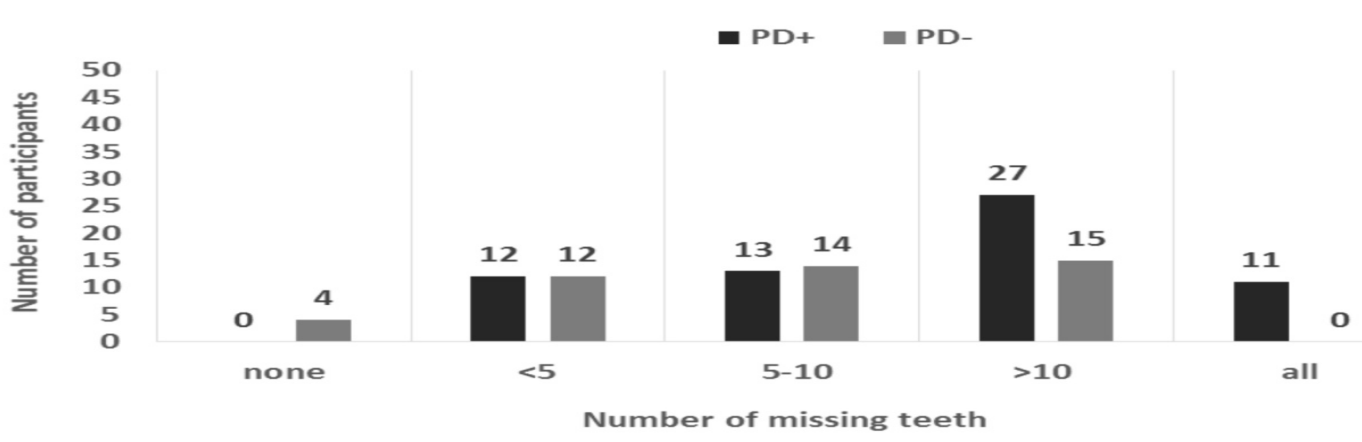

Figure 2. Number of missing teeth among the subgroups with periodontal disease present or absent.

Footnote: PD+, participants with a declared periodontal disease diagnosis present; PD-, participants without a declared periodontal disease diagnosis

Table IV. Declared cardiovascular disease (univariate analysis).

\begin{tabular}{l|c|c|c}
\hline Cardiovascular disease & $\begin{array}{c}\text { PD+ (N=63) } \\
\mathbf{\%}\end{array}$ & $\begin{array}{c}\text { PD- }(\mathbf{N}=\mathbf{4 5}) \\
\mathbf{\%}\end{array}$ & $\mathbf{p}$ \\
\hline Ischemic heart disease & 23.8 & 22.2 & $\mathbf{0 . 8 4}$ \\
\hline Angina pectoris & 7.9 & 4.4 & $\mathbf{0 . 7 0}$ \\
\hline Myocardial infarction & 9.5 & 6.7 & $\mathbf{0 . 7 3}$ \\
\hline Atherosclerotic coronary disease & 19.0 & 6.7 & $\mathbf{0 . 0 6}$ \\
\hline Stroke & 1.6 & 8.9 & $\mathbf{0 . 1 5}$ \\
\hline Cardiac failure & 7.9 & 11.1 & $\mathbf{0 . 7 3}$ \\
\hline
\end{tabular}

$\mathrm{N}$, number of participants per group; $\mathrm{PD}+$, participants with a declared periodontal disease diagnosis present; PD-, participants without a declared periodontal disease diagnosis.

\section{Discussion}

The present study assessed the correlation between periodontal disease and systemic disease and common risk factors in an urban and rural population from North-West Transylvania, Romania. We did not find in the literature another validated questionnaire used to assess these links in Romania. In our study, more than half $(58.3 \%)$ of the enrolled adults aged 45 years and older declared a diagnosis of periodontal disease. This prevalence is higher compared to the one reported in the US, where $47.2 \%$ of adults over 30 years of age were diagnosed with periodontitis, percentage that increased to $70.1 \%$ in the $\geq 65$-years age group [11].

An important finding of this study is a highly significant association between periodontal disease and a known family history of periodontal disorders, $93.5 \%$ of the participants with periodontal disease reporting family history of periodontal disorders. These results highlight the importance of the genetic factor in the etiopathogenesis of periodontal disease, being in agreement with previous findings regarding aggressive or less aggressive forms of periodontitis [12]. Also, genetics is a major risk factor in the development and progression of periodontal disease 
[13].

A higher prevalence of periodontal disease was observed in participants presenting to the dentist only for emergencies compared to participants who declared having regular dental appointments at 6 months or 1 year. These results follow the same trend as observed in a crosssectional study which enrolled 595 randomly selected participants, and found that the percentage of participants reporting emergency dental visits had a higher prevalence of periodontitis (8.3\%) compared to participants reporting regular dental visits [14].

All enrolled participants reported the presence of edentulism and all participants reporting complete edentulism had a previous declared diagnosis of periodontal disease. These results are in agreement with the present registries regarding periodontal disease as the main cause of tooth loss in adult population [15].

Although $38.1 \%$ of participants with periodontitis reported the presence of a cardiovascular disease and 36.5\% reported diabetes mellitus, we did not find a statistically significant correlation between periodontal disease and systemic diseases. Our results are similar to those observed in other studies which suggest that although periodontal disease is an independent risk factor for cardiovascular disease, there is a $24-35 \%$ higher risk of developing cardiovascular pathology among patients with periodontal disease [16].

A statistically significant correlation was observed between smoking, a common risk factor for both periodontal pathology and cardiovascular disease, and the presence of self-reported periodontal disease, similar to a great number of previous studies [17]. A healthy lifestyle which implies the intake of fresh fruit and vegetables, dairy products, as well as regular sport activities are known protective factors for cardiovascular disease [18]. We observed a low prevalence of periodontal and cardiovascular disease in participants reporting sustained physical activity. This finding is in agreement with a previous study conducted in a subset of the third national health and nutrition examination survey (NHANES III), which found that a recommended level of physical activity was associated with a lower prevalence of periodontal disease [19]. Our multivariate analysis found an association between periodontitis and atherosclerotic coronary artery disease. The findings from the literature regarding this association range from no causative relationship between periodontitis and atherosclerotic cardiovascular disease and a strong connection between the two conditions, mainly due to variations in the study populations and different definitions and measures of periodontal disease $[7,20]$.

The present study found a slightly higher prevalence of self-reported periodontal disease among female participants compared to men. This high prevalence in women could be due to the fact that they are more interested in aesthetic and functional disturbances compared to men, therefore more women than men present to the dentist when these requirements are not accomplished. The prevalence of self-reported periodontal disease was slightly higher in the rural population compared to the urban population $(50.9 \%$ versus $49.1 \%$ ), probably due to poorer socio-economic conditions and reduced accessibility to healthcare services compared to the urban area. However, using the data from the third National Health and Nutrition Examination Survey (NHANES III), Borrell and co-workers found an association between periodontitis and the demographic parameters of the studied population [21].

One limitation of our study is the small sample analyzed. Additionally, being a questionnaire-based study, the majority of the investigated variables are based on the answers provided by the participants and in some cases these answers might not be accurate. Nevertheless, the developed questionnaire was validated and can be used in larger scale studies to assess the link between periodontitis and systemic diseases. The questionnaire could also be used for the early identification of the risk factors and implementation of the primary preventive measures, an important feature of public health programs.

\section{Conclusions}

Our study found a statistically significant association between periodontal disease and a known family history of periodontal disorders and smoking duration. Daily intake of fresh fruit and increased sport activities were found to be protective factors against periodontal disease. Periodontitis was found as a risk factor for atherosclerotic cardiovascular disease. Further research is needed to identify the significant correlations between periodontal disease and systemic disorders and to establish a causality between them, with an impact on the primary prevention methods.

\section{Acknowledgments}

The authors would like to thank the participants who took part in this study. The authors acknowledge the input of the experts who contributed to the development of the questionnaire. Also, the authors thank the study investigators who administered the questionnaires and the participants who evaluated the questionnaires as part of the pilot study.

\section{References}

1. Pihlstrom BL, Michalowicz BS, Johnson NW. Periodontal diseases. Lancet. 2005;366:1809-1820.

2. Carramolino-Cuéllar E, Tomás I, Jiménez-Soriano Y. Relationship between the oral cavity and cardiovascular diseases and metabolic syndrome. Med Oral Patol Oral Cir Bucal. 2014;19:e289-e294.

3. Gurav AN. The association of periodontitis and metabolic syndrome. Dent Res J. 2014;11:1-10.

4. Nagpal R, Yamashiro Y, Izumi Y. The Two-Way Association of Periodontal Infection with Systemic Disorders: An Overview. Mediators Inflamm. 2015;2015:793898. doi: 
$10.1155 / 2015 / 793898$.

5. Arigbede AO, Babatope BO, Bamidele MK. Periodontitis and systemic diseases: A literature review. J Indian Soc Periodontol. 2012;16:487-491.

6. Linden GJ, Lyons A, Scannapieco FA. Periodontal systemic associations: review of the evidence. J Clin Periodontol. 2013;40 Suppl 14:S8-S19.

7. Friedewald VE, Kornman KS, Beck JD, Genco R, Goldfine A, Libby P, et al. The American Journal of Cardiology and Journal of Periodontology Editors' Consensus: periodontitis and atherosclerotic cardiovascular disease. Am J Cardiol. 2009;104:59-68.

8. Lalla E, Papapanou PN. Diabetes mellitus and periodontitis: a tale of two common interrelated diseases. Nat Rev Endocrinol. 2011;7:738-748.

9. Preshaw PM, Alba AL, Herrera D, Jepsen S, Konstantinidis A, Makrilakis K, et al. Periodontitis and diabetes: a two-way relationship. Diabetologia. 2012;55:21-31.

10. Suvan J, D'Aiuto F, Moles DR, Petrie A, Donos N. Association between overweight/obesity and periodontitis in adults. A systematic review. Obes Rev. 2011;12:e381-404.

11. Eke PI, Dye BA, Wei L, Thornton-Evans GO, Genco RJ; CDC Periodontal Disease Surveillance workgroup: James Beck (University of North Carolina, Chapel Hill, USA), Gordon Douglass (Past President, American Academy of Periodontology), Roy Page (University of Washington. Prevalence of periodontitis in adults in the United States: 2009 and 2010. J Dent Res. 2012;91:914-920.

12. AlJehani YA. Risk factors of periodontal disease: review of the literature. Int J Dent. 2014;2014:182513. doi: $10.1155 / 2014 / 182513$.

13. Quappe L, Jara L, Lopez NJ. Association of interleukin-1 polymorphisms with aggressive periodontitis. J Periodontol. 2004;75:1509-1515.
14. Ababneh KT, Abu Hwaij ZM, Khader YS. Prevalence and risk indicators of gingivitis and periodontitis in a multi-centre study in North Jordan: a cross sectional study. BMC Oral Health. 2012;12:1. doi: 10.1186/1472-6831-12-1.

15. Al-Harthi LS, Cullinan MP, Leichter JW, Thomson WM. The impact of periodontitis on oral health-related quality of life: a review of the evidence from observational studies. Aust Dent J. 2013;58(3):274-277.

16. Humphrey LL, Fu R, Buckley DI, Freeman M, Helfand M. Periodontal disease and coronary heart disease incidence: a systematic review and meta-analysis. J Gen Intern Med. 2008;12:2079-2086.

17. Stabholz A, Soskolne WA, Shapira L. Genetic and environmental risk factors for chronic periodontitis and aggressive periodontitis. Periodontol 2000. 2010;53:138-153.

18. Perk J, Backer G, Gohlke H, Graham I, Reiner Z, Verschuren WM, et al. European Guidelines on cardiovascular disease prevention in clinical practice (version 2012): the Fifth Joint Task Force of the European Society of Cardiology and Other Societies on Cardiovascular Disease Prevention in Clinical Practice (constituted by representatives of nine societies and by invited experts). Eur J Prev Cardiol. 2012;19:585-667.

19. Al-Zahrani MS, Borawski EA, Bissada NF. Increased physical activity reduces prevalence of periodontitis. J Dent. 2005;33:703710.

20. Rydén L, Buhlin K, Ekstrand E, de Faire U, Gustafsson A, Holmer J, et al. Periodontitis Increases the Risk of a First Myocardial Infarction: A Report From the PAROKRANK Study. Circulation 2016;133(6):576-583.

21. Borrell LN, Burt BA, Warren RC, Neighbors HW. The role of individual and neighborhood social factors on periodontitis: the third National Health and Nutrition Examination Survey. J Periodontol. 2006;77:444-453. 